\title{
The Age of Data: pinpointing guilty bytes in polymorphic buffer overflows on heap or stack
}

\author{
Asia Slowinska, Herbert Bos \\ Vrije Universiteit Amsterdam \\ Department of Computer Science, Faculteit der Exacte Wetenschappen \\ De Boelelaan 1081, 1081 HV Amsterdam, Netherlands \\ $\{$ asia, herbertb\}@few.vu.nl
}

\begin{abstract}
Heap and stack buffer overflows are still among the most common attack vectors in intrusion attempts. In this paper, we ask a simple question that is surprisingly difficult to answer: which bytes contributed to the overflow? By careful observation of all scenarios that may occur in overflows, we identified the information that needs to be tracked to pinpoint the offending bytes. There are many reasons why this is a hard problem. For instance, by the time an overflow is detected some of the bytes may already have been overwritten, creating gaps. Additionally, it is hard to tell the offending bytes apart from unrelated network data. In our solution, we tag data from the network with an age stamp whenever it is written to a buffer. Doing so allows us to distinguish between different bytes and ignore gaps, and provide precise analysis of the offending bytes. By tracing these bytes to protocol fields, we obtain accurate signatures that cater to polymorphic attacks.
\end{abstract}

Keywords: attack analysis, intrusion detection and prevention, honeypots

\section{Introduction}

Polymorphic network attacks are difficult to detect and even harder to fingerprint and stop. This is especially true if the exploit itself is polymorphic [11]. We define fingerprinting as the process of finding out how an attack works. It is important for two reasons: analysis of the attack (e.g., by human security experts), and signature generation.

Signature generation is hard because of the complex and conflicting list of constraints. Signatures should incur a negligible ratio of false positives, while the num- ber of false negatives should be low. Also, we should be able to check signatures at high rates and cater to polymorphic attacks with polymorphic exploits. We further aim for fast, one-shot generation without the need to replay the attack.

In this paper, we address the problem of polymorphic buffer overflow attacks on heap and stack. Given their long history and the wealth of counter-measures, it is perhaps surprising that buffer overflows are still the most popular attack vector. For instance, more than one third of all vulnerabilities notes reported by US-CERT in 2006 consisted of buffer overflows [31]. As the US-CERT's database contains many types of vulnerabilities (leading to denial of service, privacy violation, malfunctioning, etc.), the percentage of buffer overflows in the set of vulnerabilities leading to control over the victim is likely to be higher. Even Windows Vista, a new OS with overflow protection built into the core of the system, has shown to be vulnerable to such attacks [25].

Polymorphic attacks demand that signature generators take into account properties other than simple byte patterns. For instance, previous approaches have examined such properties as the structure of executable data [14], or anomalies in process/network behavior $[9,15,17]$.

In contrast, in this work we asked a simple question that is surprisingly hard to answer: what bytes contribute to an attack? As we will see, an answer to this question also trivially yields reliable signatures. Like [4], we focus on vulnerabilities rather than specific attacks, which makes the signatures impervious to polymorphism. However, besides signatures, we believe the answer to the above question is invaluable for later analysis by human experts.

The full system is known as Prospector, a protocolspecific detector of polymorphic buffer overflows. It 
deals with both heap and stack overflows in either the kernel or user processes and while it was implemented and evaluated on Linux, the techniques apply to other OSs also.

In a nutshell, the idea is as follows (see also Figure 1). We use an emulator-based honeypot with dynamic taint analysis [24] to detect attacks and to locate both the exact address where a control flow diversion occurs and all the memory blocks that originate in the network (known as the tainted bytes). The emulator marks all bytes originating in the network as tainted, and whenever the bytes are copied to memory or registers, the new location is tainted also. We trigger an alert whenever the use of such data violates security policies.

Next, we track which of the tainted bytes took part in the attack. For instance, in a stack overflow we walk up the stack looking for tainted bytes. However, we must weed out all the memory that, while tainted, had nothing to do with the attack (e.g., stale data that was part of an old stack frame, such as the bytes marked $x$ in the figure). To do so, we track the age of data at runtime, so that we know whether memory on the heap or stack is a left-over from an older allocation and can distinguish relevant bytes from memory to be ignored.

Once we know which bytes were in the buffer overflow and we can trace them to the bytes that arrived from the network, we find out which protocol fields contributed to the attack. If $n$ fields were involved in the overflow with a combined length of $N$, we know that any similar protocol message with a combined length for these fields greater or equal to $N$ will also lead to a buffer overflow.

Contributions. Our main contribution is the identification of all bytes contributing to an overflow. The identification is performed in a single interaction (i.e., without need for replaying attacks) and is sufficiently fast to be used in honeypots. The signature generator is intended to demonstrate the usefulness of such data in practice. While the end result is a powerful signature generator in its own right, very different signature generators could also be built on this technique. For instance, we essentially yield snort-like patterns which may be used if the attack is not polymorphic. In addition, it could generate a wealth of information for human security experts.

A second contribution is that we extend taint analysis in the temporal domain. In its simplest form, taint analysis is zero-dimensional and consists of a single bit for every data item to indicate whether or not it originates in a suspect source. More advanced analysis extends the analysis in the spatial dimension, by tracking exactly where the data originated (e.g., Vigilante and
Argos both maintain a pointer in the network trace). In this paper, we extend tracking in the temporal domain by storing when data is tainted. We argue that this is essential information for signature generators that allows us to separate relevant bytes from unrelated tainted memory.

A third contribution is that we first show that wellknown existing vulnerability-based signatures based on the length of a protocol-field (e.g., Covers [16]) are weak and frequently incur both false positives and false negatives, and then remedy the weakness so as to make false positives virtually impossible and false negatives implausible.

A fourth contribution is that we extend the vulnerability signatures to include attacks based on protocol messages that contain a specially forged (wrong) length field. For instance, such fields specify the length of another protocol field and by providing a wrong value, the attack coerces vulnerable programs into allocating buffers that are too small and that overflow when the actual data exceeds the specified length. We will discuss more advanced attacks of this type also. Few existing projects address such attacks.

Finally, we extended Prospector with an attack vector-specific module to make it deal with double free attacks.

Outline. Section 2 discusses related work. Section 3 highlights factors in heap and stack overflows that complicate the analysis. Sections 4 and 5 describe the design and implementation of Prospector, respectively. Prospector is evaluated in Section 6. Conclusions are drawn in Section 7.

\section{Related work}

Previous work on detection of polymorphic attacks focused on techniques that look for executable code in messages, including: (a) abstract or actual execution of network data in an attempt to determine the maximum executable length of the payload [30], (b) static analysis to detect exploit code [5], (c) sled detection [1], and (d) structural analysis of binaries to find similarities between worm instances [14].

Taint-analysis is used in several projects for signature generation $[21,7]$. However, none of these projects provide an answer to the question of which bytes were involved. Enhanced tainting [3] expands the scope of tainting to also detect such attacks as SQL injection and XSS, but requires source code transformation.

Transport-layer filters independent of exploit code are proposed in Shield [32] with signatures in the form of partial state machines modeling the vulnerability. Specific protection against instruction and register 


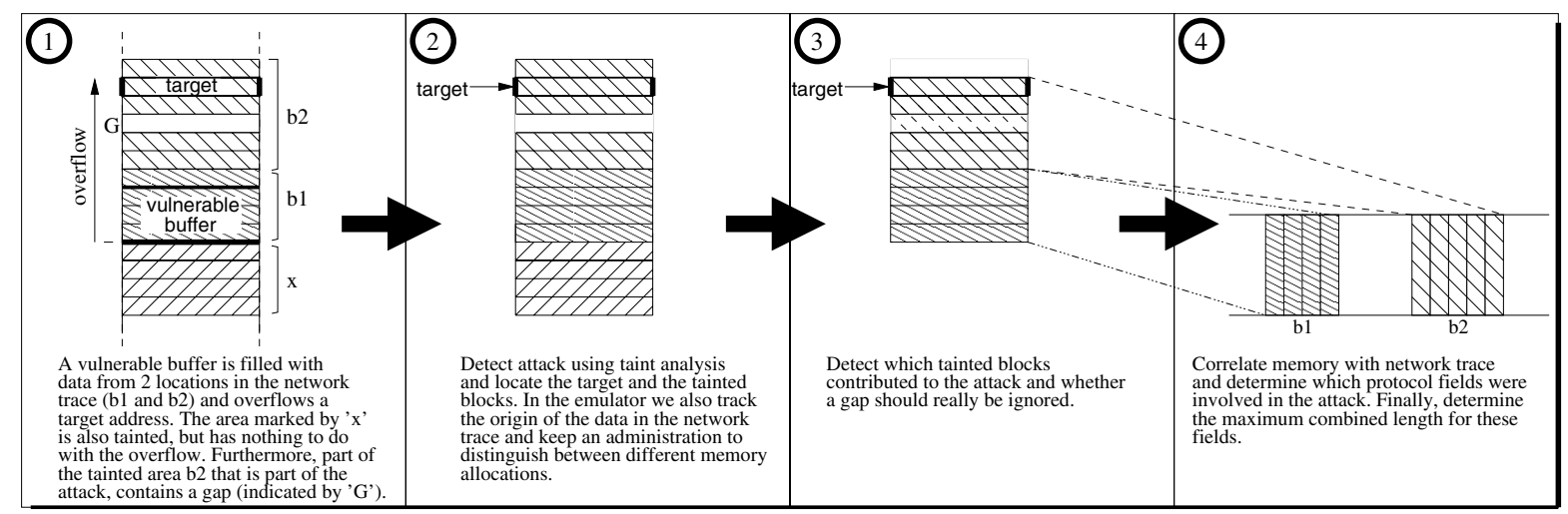

Figure 1. Main steps in Prospector's attack analysis.

shuffling, as well as against garbage insertion is offered by semantics-aware detection [6].

A related project, PolyGraph [20], fingerprints attacks by looking at invariant substrings present in different instances of suspicious traffic. The idea is to use these substrings as a signature. Such methods are vulnerable to the injection of noise in the data stream [23].

Various groups have proposed anomaly detection for catching polymorphic attacks. PAYL [33] builds a model of the byte distribution of normal traffic and compares real traffic with this model. Increasingly sophisticated mimicry attacks $[13,12]$ are a problem and spark many new developments in this direction $[10,9]$.

SigFree [35] observes that overflow attacks typically contain executables whereas legitimate requests never contain executables, and blocks attacks by detecting the presence of code.

Brumley et al. propose vulnerability-based signatures [4] that match a set of inputs (strings) satisfying a vulnerability condition (a specification of a particular type of program bug) in the program. When furnished with the program, the exploit string, a vulnerability condition, and the execution trace, the analysis creates the vulnerability signature for different representations, Turing machine, symbolic constraint, and regular expression signatures.

Packet Vaccine [34] detects anomalous payloads, e.g., a byte sequence resembling a jump address, and randomizes it. Thus, exploits trigger an exception in a vulnerable program. Next, it finds out information about the attack (e.g., the corrupted pointer and its location in the packet), and generates a signature, which can be either based on determination of the roles played by individual bytes, or it can be much like Covers [16]. In the former case, Packet Vaccine scrambles individual bytes in an effort to identify the essential inputs. In the latter case, the engine finds the field containing the jump address and estimate the length needed to cause an overflow. These coarse signatures are subse- quently refined by trying variations of the vaccine, that is, the engine iteratively alters the size of the crucial field, and checks for the program exception. Packet Vaccine suffers from the same problems as Covers. It neither checks for multiple separate fields, nor worries about the granularity of the protocol dissector. Also, it does not address the problem of attacks based on malformed length fields mentioned earlier. By passing over these issues, this approach may lead to false negatives and positives.

\section{Overflow attacks}

Prospector caters to both heap and stack overflows. Stack overflows are conceptually simple. Even so, they prove to be hard to analyze automatically. Essentially, a vulnerable buffer on the stack is overflown with network data until it overwrites a target that may lead to control flow diversion (typically the return address). Observe that the data that is used for the overflow may originate in more than one set of bytes in the network flow (examples in practice include the well-known Apache-Knacker exploit [28]). In Figure 1 this is illustrated by regions $b 1$ and $b 2$. Taking into account either fewer or more protocol fields may lead both to false positives and negatives. Covers [16], by using a single protocol field, lacks accuracy in a multi-field attack.

There is another, more subtle reason why this may occur, even if the attack does not use multiple fields: the protocol dissector used to generate signatures may work at different protocol field granularities than the application. For instance, the dissector may identify subfields in a record-like protocol field as separate fields, while the application simply treats it a single protocol field. As a consequence, the two types of misclassification described above may occur even for 'single-field' exploits. As we often do not have detailed information about the application, this scenario is quite likely. Again, solving the problem requires handling 
'multi-field' attacks properly.

Gaps. The naive solution for finding the bytes that contribute to the attack is to start at the point of attack (the target in Figure 1) and grab every tainted byte below that address until we hit a non-tainted byte. Unfortunately, while all bytes that contributed to the attack were tainted at some point, such a naive solution is really not adequate. First, there may be gaps in the tainted block of memory that was used in the attack. For instance, the code in Listing 1 may lead to a gap, because the assignment to $n$ occurs after the overflow.

Unrelated taints. Second, the naive solution gathers tainted blocks that are unrelated to the attack. An example is the region marked by $x$ in Figure 1. It may be caused by left-over data tainted from an old stack frame, or by safe buffers adjacent to the vulnerable buffer, such as the buffer unrelated in Listing 1. In this paper, we will informally refer to such unrelated tainted data as unrelated taints.

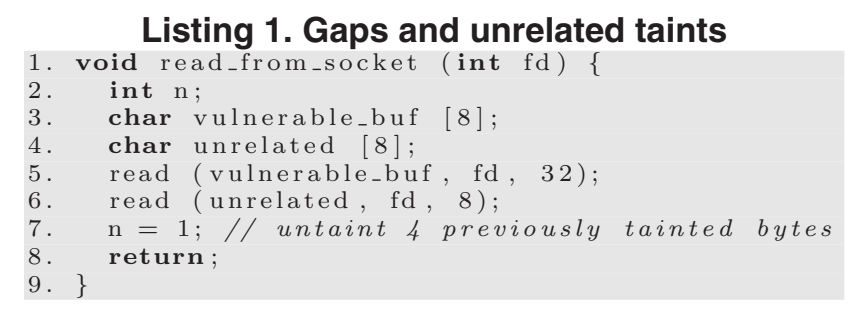

Heap corruption can be more complex than a stack overflow and potentially more powerful. A simple overflow occurs when critical data (e.g., a function pointer) is overwritten from a neighboring chunk of memory, or from another field of a structure. In a more advanced form, the attacker overflows link pointers that are used to maintain a structure keeping free regions. It allows an attacker to overwrite virtually any memory location with any data [2]. The problem is caused by the implementation of memory allocation functions which store control data together with the actual allocated memory, thus providing attackers potential access to information used by the operating system memory management.

The problem of gaps and unrelated taints also exists for heaps and is mostly similar to that of the stack. For heap overflows, instead of the occurrence of stale tainted data from a previous function call, we may encounter stale tainted data used in a previous function that allocated the memory region. In addition, there may be taints in adjacent fields of a structure. Advanced heap corruption attacks yield an additional complication. Since the attacker can overwrite any memory location with any contents, it is possible that at detection time the memory region which was holding the vulnerable buffer is reused and contains unrelated data. If left unhandled, such a scenario would prevent us from pin-pointing exactly the data responsible for the intrusion attempt.

Length field attacks. Finally, numerous protocols have fields specifying the length of another field, say $l_{f}$ defining the length of field $f$. Attackers may manipulate this length value, and via heap overflows take control of the host. First, a malicious message may provide $l_{1}$, with $l_{1} \gg l_{f}$ and close to the maximum size of an integer. The application allocates $l=l_{1}+k$ bytes (where $k$ bytes are needed to store some applicationspecific data), and $l$ ends up being a small number because of the integer wrap-around, $l \ll l_{1}$. As a result, the application copies $l_{1}$ bytes into the buffer leading to overflow. In a second scenario, rarely seen in the wild, the attacker provides $l_{2}$ smaller than expected, $l_{2}<l_{f}$, the application allocates a buffer of size $l_{2}$ which is not sufficient to hold the data, and a subsequent copy operation without boundary checks spills network data over adjacent memory. Notice that we cannot draw any conclusions about a message containing such attacks by relying only on the observation that $n$ fields where involved with a combined length of $N$.

We conclude this section with the assumption that overflows occur by writing bytes beyond the high end of the buffer, since it makes the explanation easier. However, it is trivial to extend our techniques to handle the reverse direction (attacks overwriting memory below the start of a buffer).

\section{Design}

The main steps of Prospector's attack analysis are sketched in Figure 1. In this section, we first describe how we instrument the execution and what data is produced by our taint-analysis emulator. We then show how we use this data to determine the exact bytes in the attack. The memory that constitutes these bytes will be referred to as the crucial region. Finally, we correlate the information with protocol fields in network data to obtain signatures.

\subsection{Dynamic taint analysis}

Prospector employs an efficient and reliable hardware emulator that uses taint analysis to tag and track network data [24]. Data originating in the network is marked as tainted, and whenever it is copied to memory or registers, the new location is tainted also. We raise an alert whenever the use of such data violates security policies. To aid signature generation we dump the content of all registers, as well as tainted memory 
blocks to file, with markers specifying the address that triggered the violation, the memory area it was pointing to, etc. In addition, we keep track of the exact origin of a tainted memory area, in the form of an offset from the start of the network trace. In practice, the offset is used as (32 bit) tag.

Even with such accurate administration of offsets, the problem of identifying crucial regions remains. We therefore extended the tracking in the temporal domain. In the next few sections we will explain the blocks that together form our information correlation engine. We start with support for an advanced heap corruption attack, and then explain how we pinpoint the relevant tainted memory region.

\subsection{Dealing with advanced heap overflows}

In the case of stack overflows and simple heap corruption attacks, we know from where to look for the crucial regions: in the memory area beneath the violation address reported by the emulator. In contrast, advanced heap corruption attacks, require us to find first the memory region containing the vulnerable buffer. Only then we can start marking the bytes that contributed to the attack.

Such attacks may easily lead to a situation in which at detection time, the memory region that was holding the vulnerable buffer is reused and contains unrelated data. Prospector therefore marks the bytes surrounding an allocated chunk of memory as red. When tainted data that is written to a red region (representing an overflow, but not necessarily an attack), we keep the application running, but dump the memory region covering the whole vulnerable buffer for potential later use. This works as common memory management systems store control data in-line together with allocated chunks of memory. Consequently, the 'red' bytes surrounding an allocated buffer contain control data, which should never be overwritten with data coming from the network.

In the case of an intrusion attempt, we search for the violation address and the network index in the dumped heap areas in order to find a memory region containing the buffer that contributed to the attack. These chunks of memory allow us to perform further analysis described in Section 4.8. Note that red markers are quite different from StackGuard's canary values [8], as they are maintained by the emulator and trigger action immediately when they are overwritten.

\subsection{Dealing with malformed messages}

To handle heap corruption attacks that use malformed length fields, we check whether allocating a chunk of memory relies on remote data. Whenever an application calls malloc (size) with the size variable being tainted, we associate the network origins of the length parameter with the new memory chunk. In the case of an intrusion attempt, it enables us to determine the real cause, and generate a correct signature. For details, see Section 4.9.1.

\subsection{Age stamps}

In order to distinguish between stale and relevant data both on stack and heap we introduce an age stamp indicating the relative age of data regions. AgeStamp is a global counter, common to the entire OS running on the emulator. The need for a system-wide global variable stems from the fact that memory may be shared.

AgeStamp is increased whenever a function is called (a new stack frame is allocated) or returns. To be precise, we update AgeStamp $v_{1}$ to $\left(v_{1}+1\right)$ only if in epoch $v_{1}$ a tainted value was stored in the memory. Otherwise it is not necessary, as we shall soon see. If a tainted value is copied to memory, we associate the current AgeStamp with the destination memory location, i.e., for each tainted value we remember the epoch in which it was stored. In addition, for each process and lightweight process we allocate a history buffer, where we store information about allocation and release of stack frames, as follows: for each function call and return we store the value pair (stack pointer, AgeStamp). When an application allocates a buffer on the heap, we associate the current AgeStamp with this memory region. When a memory field becomes untainted, we do not clean the age stamp value.

We observe that the order of age stamps in the crucial region right after the overflow (before gaps appear) is nondecreasing. We will use this observation in the analysis phase to spot tainted bytes stored later than the crucial tainted memory region, forming either a gap, or an area of unrelated taints. For instance, the unrelated buffer in Listing 1 has age stamps greater than vuln_buf, and so we can conclude that it does not belong to the crucial memory region.

\subsection{Additional indicators}

Even though age stamps provide a crude separation of unrelated taints, they are not powerful enough. Let us consider an example vulnerable a_fun function in Figure 2. For simplicity we discuss a stack example, 


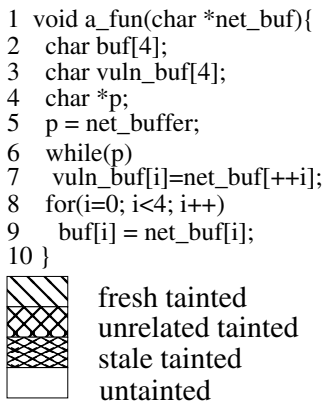

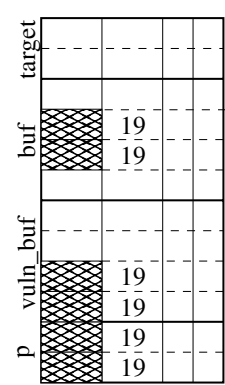

(a)

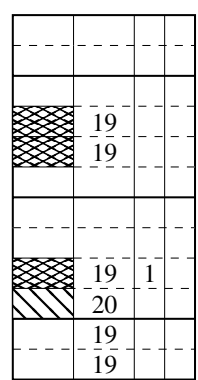

(b)

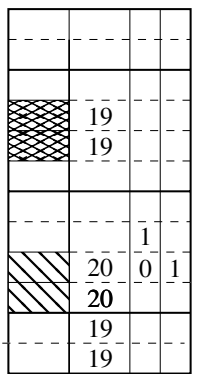

(c)

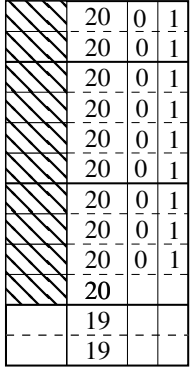

(d)

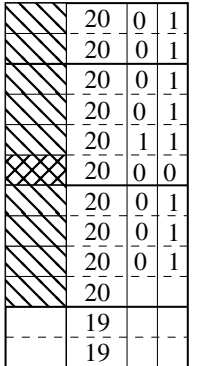

(e)

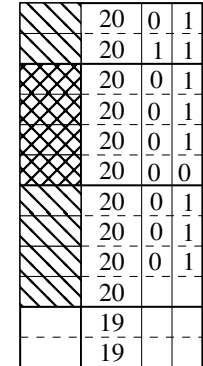

(f)

(a) memory allocation, AgeStamp=20; (b) assignment in line 5, and first iteration of the while loop, (c) second iteration of the while loop, (d) remaining iterations of the while loop, (e) first iteration of the for loop, (f) remaining iterations of the for loop Since $\mathrm{p}$ is untainted, and we cannot deduce values of its associated indicators, they are left blank.

Figure 2. A series of the emulator's memory maps presenting values associated with local variables

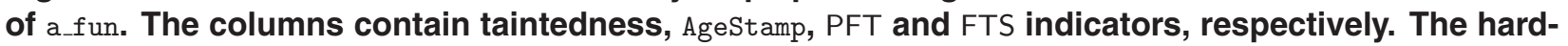
ware emulator cannot certainly distinguish between stale, fresh and unrelated tainted bytes. It just says whether a memory location is tainted or not. The figure contains various patterns for clarity reasons.

but the method is used for the heap also. The figure illustrates a series $(a-f)$ of the emulator's memory maps presenting values associated with local variables of a_fun. For now, we limit our interest to the first two columns containing information about taintedness and AgeStamp, respectively.

We assume that the example function is executed in an epoch with AgeStamp equal to 20, so that the few existing tainted bytes with AgeStamp 19 are stale. Note that by using while and for loops, a_fun copies network data without any calls and thus without incrementing AgeStamp. Even though we duly raise an alert after step $2 f$, when the function returns and is about to jump to an address influenced by the attacker, the memory dump and the age stamps do not provide the means to separate the relevant bytes from the unrelated buffer buf. The reason is that vuln_buf and buf have the same AgeStamp.

To remedy this situation we introduce two extra 1-bit indicators for each memory location to let us establish the order in which the buffers were filled: PFT (Previous address Freshly Tainted) and FTS (First Tainted Store), respectively. Intuitively, PFT indicates for address $a$ whether $a-1$ was assigned fresh tainted contents. If $a$ is tainted, then PFT signifies that the contents of $a-1$ is more recent than that of $a$. The FTS bit indicates that the tainted store at address $a$ was the first such store to $a$ after $a-1$ was tainted. However, their exact meanings are defined by the algorithm in Listing 2. As the semantics of these two additional indicators are complex, we introduce them by way of a detailed example.

\subsection{Example explained}

We return to the example in Figure 2 and examine values of PFT and FTS, i.e., the values in the last two columns of the memory maps.

The assignment operation in line 5 sets memory associated with $\mathrm{p}$ as untainted, and leaves Prospector's markers untouched (Figure 2b).

This brings us to the execution of the while loop in lines 6-7. The first iteration marks addr vuln_buf $_{\text {tainted, }}$ sets AgeStamp(addr $r_{v u l n \_b u f}$ ) to the current value of AgeStamp, and PFT( $\left.\operatorname{addr}_{\text {vuln_buf }}+1\right)$ to 1 . We informally interpret it as addr $v_{v u l n \_b u f}$ telling (addr $\left.v_{v u l n \_b u f}+1\right)$ : "I have tainted contents, more fresh than yours". We still need to decide about FTS( $\left.\operatorname{addr}_{v u l n \_b u f}\right)$. As we do not know the value of PFT( $\left.\operatorname{addr}_{v u l n \_b u f}\right)$, let us assume, for example, that PFT is unset. In this case, addr $v_{v u l n \_b u f}$ has already 'consumed the message' from ( addr $_{v u l n \_b u f}$ 1 ), and so the current store operation is not the first since ( $\operatorname{addr}_{\text {vuln_buf }}-1$ ) became tainted. We record this information by unsetting FTS( $\left.\operatorname{addr}_{\text {vuln_buf }}\right)$.

Figure 2c presents the second iteration of the while loop in lines 6-7. We mark addr $v_{v u l n \_b u f}+1$ as tainted, set AgeStamp (addr $\left.r_{v u l n \_b u f}+1\right)$ to the current value of AgeStamp, and PFT( $\operatorname{addr}_{\text {vuln_buf }}+2$ ) to 1, thus informing the memory location above it that $\operatorname{addr}_{v u l n \_b u f}+1$ has freshly tainted contents. This time we know that no tainted store operation was executed since addr $v_{v u l n \_b u f}$ became tainted. We set FTS $\left(\operatorname{addr}_{\text {vuln_buf }}+1\right)$ to 1 , and also unset PFT( $\left.\operatorname{addr}_{v u l n \_b u f}+1\right)$, since the tainted value of $\operatorname{addr}_{\text {vuln_buf }}+1$ is more recent than that of $\operatorname{addr}_{\text {vuln_buf }}$.

Figure $2 \mathrm{~d}$ illustrates the memory map just after the while loop. Observe that all bytes inside the tainted 
Listing 2. Algorithm for updating the indicators

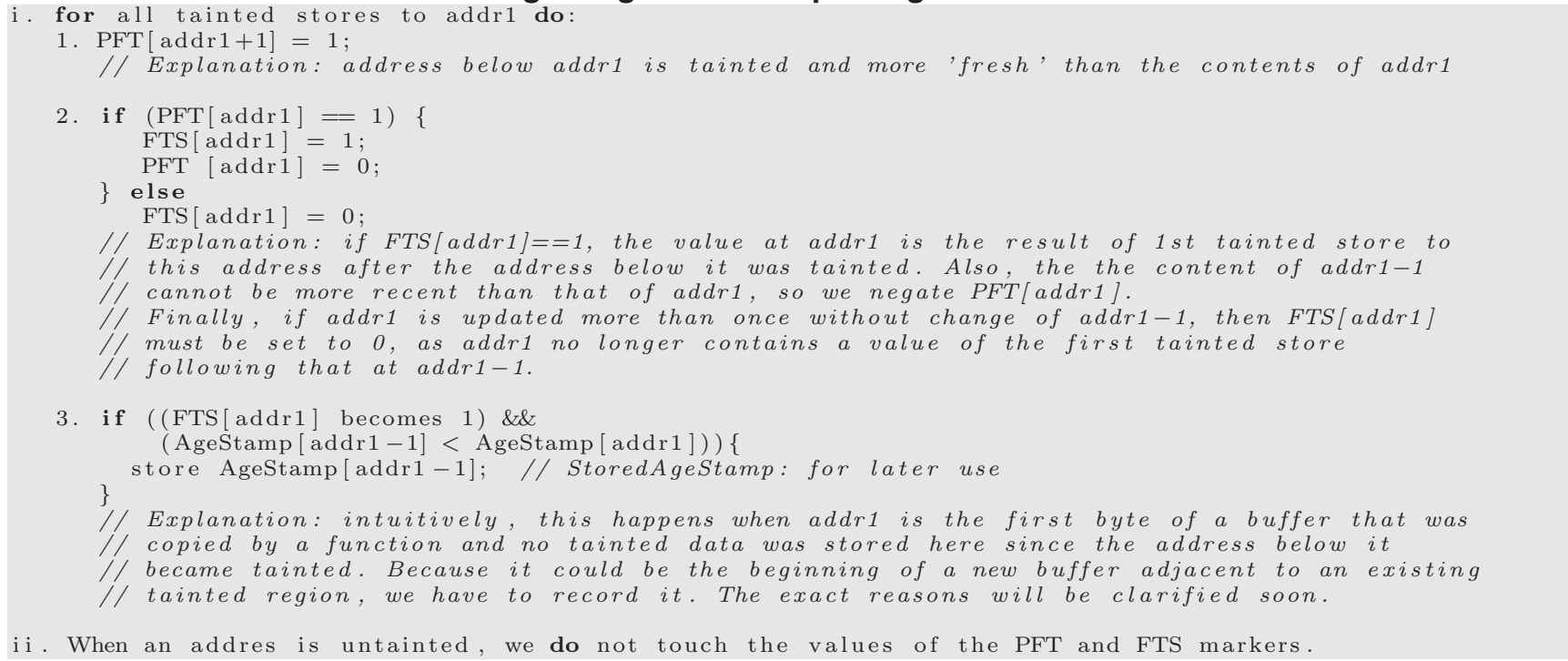

memory region which contributed to the attack have PFT unset, and FTS set to 1.

This brings us to the for loop in lines 8-9, the first iteration of which can be examined in Figure 2e. While storing the first byte in the gap, we set PFT $\left(\operatorname{addr}_{b u f}+1\right)$ to 1 , and also check that the current store operation is not the first one since addr $r_{b u f}-1$ became tainted. Indeed, the assignment in the fifth iteration of the while loop held this property. So, we unset FTS( $\operatorname{addr}_{b u f}$ ).

Finally, Figure $2 \mathrm{f}$ presents the whole gap formed by buf. Observe that the gap internally has PFT negated, and FTS set, just like a 'typical' tainted region. However, the byte just above the gap has PFT set to 1 , as a result of the store in the fourth iteration of the for loop. In that iteration, $\left(\operatorname{addr}_{b u f}+3\right)$ informed the memory location above it about its freshly tainted contents. Since this was the last byte of the unrelated buffer, no store operation has 'consumed this message'. Similarly, the bottom byte of the gap has both indicators negated.

Now that we have an intuitive grasp of the use of the additional indicators, we are ready to turn to more formal definitions (Section 4.7) and analysis (Section 4.8).

\subsection{Formal specification of properties of tainted data and gaps}

In this section, we use the indicators defined above to derive properties of regions of tainted memory.

Observation 1 Let buf be a crucial tainted region of size $n$. Then:

(a) $\forall i=0 \ldots(n-1)$ : buf $[i]$ is tainted,

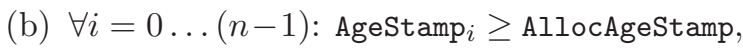
where AllocAgeStamp is the epoch in which the buffer was allocated,

(c) $\forall i, j=0 \ldots(n-1), i<j: \operatorname{AgeStamp}_{i} \leq$ AgeStamp $_{j}$

(d) $\forall i=1 \ldots(n-1): \operatorname{PFT}(\operatorname{buf}[i])$ is unset, and FTS (buf $[i]$ ) is set (as the store at buf $[i]$ finds PFT set).

Observation 2 Let gap be a non-tainted discontinuity located inside a crucial tainted memory region buf, i.e., a region in buf where Observation 1.a does not hold. Since neither age stamps nor indicators are changed when a memory location becomes untainted, Observations 1.b-1.d also hold within gap.

Observation 3 Let gap be a tainted discontinuity of size $m$ inside a crucial tainted memory region buf. Then:

(a) $\forall i=0 \ldots(m-1) \operatorname{gap}[i]$ is tainted,

(b) $\forall i=0 \ldots(m-1)$ AgeStamp $(\operatorname{gap}[i]) \geq$ AgeStamp (gap $[m])^{1}$.

(c) gap $[m]$ has both indicators PFT and FTS set to 1 , gap [0] has both indicators set to 0 .

(d) $\forall i=1 \ldots(m-1): \operatorname{PFT}(\operatorname{gap}[i])$ is unset, and $\mathrm{FTS}(\operatorname{gap}[i])$ is set.

A gap containing unrelated taints may adjoin a similar gap. In that case, they simply merge as follows.

\footnotetext{
${ }^{1}$ While gap has only $m$ bytes and gap $[m]$ strictly speaking does not exist, we use it as a C-like shorthand for 'the byte above gap'.
} 
If gap is a tainted discontinuity located inside a crucial region buf, and the bottom (top) part of gap adjoins another tainted discontinuity $\operatorname{gap}_{b}\left(\operatorname{gap}_{t}\right)$, then both holes merge together forming a single discontinuity for which all properties listed in Observation 3 hold.

\subsection{Analysis: pinpointing the bytes re- sponsible for the overflow}

To find the bytes that contributed to the attack (the crucial region), we traverse the memory downwards starting at the violation address and continue as long as the bytes we come across conform to Observation 1 . In this Section we discuss how to start this process and how to overcome the complicating factors mentioned in Section 3.

\subsubsection{The age of allocation}

We start the analysis by figuring out AllocAgeStamp, the age (or epoch) in which the vulnerable buffer containing the violation address was allocated. We need it to distinguish between fresh and stale data.

In the case of a heap corruption attack, the age stamp was explicitly maintained for each chunk of memory. In a stack smashing attack (i.e., when the violation address is not smaller than the value of the stack pointer register ESP), we check the history of stack frames associated with the vulnerable process for the most recent entry above the violation address. If the malicious data was spilled over the adjacent stack frame as well, we may find an age stamp of a caller function instead. However this does not prevent the correct analysis, because when we start looking for the whole crucial region later, we will figure out the most recent, and proper AgeStamp.

\subsubsection{Gaps}

One of the difficulties identified in Section 3 concerned gaps in the crucial region's tainted data. As we have seen, such discontinuities may occur for instance when the program assigns a new value to a local variable allocated in the crucial region after the overflow took place. They can also arise if parts of the vulnerable buffer are refilled by the application. Let us assume for now that the discontinuity is fully included in the crucial tainted memory region, i.e., below the gap there is at least one byte which contributed to the attack.

Again, to find the crucial region we traverse the memory as long as the bytes encountered are in accordance with Observation 1. However, we now come across a discontinuity before we reach the region's bottom. To handle such gaps, we look for the end of the discontinuity to find out how many bytes of the crucial region we are missing. In the following, assume that add $r_{1}$ is a memory location at variance with at least one of the properties of Observation 1.

If we find a byte at variance only with Observation 1.a (i.e., it is not tainted), we conclude that it belongs to a non-tainted discontinuity. We traverse the memory further until we encounter tainted data. Since we assume that the gap does not reach the beginning of the vulnerable buffer, we will find a tainted byte.

We can also find a byte in memory location addr 1 at variance with Observation 1.d. This means that the values of indicators are corrupted: $\mathrm{PFT}\left(\operatorname{addr}_{1}\right)$ is not equal to 0 and/or FTS $\left(\operatorname{addr}_{1}\right)$ is not equal to 1 . If both indicators are set to one, then the memory location below has freshly tainted contents. Observation 3, defining gaps, says that it is probable that we have just spotted a tainted discontinuity. We now traverse the memory until we encounter a memory location with the two indicators not set. Let us now assume that the inconsistency with Observation 1.d means that FTS( $\left.\operatorname{addr}_{1}\right)$ is equal to 0 . At first sight, one may think that a new tainted store operation at $\operatorname{addr}_{1}$ caused the change of the indicator, but then the memory location above it $\left(\operatorname{add} r_{1}+1\right)$ would have PFT set to 1 , which would also have conflicted with Observation 1.d. As we did not detect this, such a case will not occur.

Similar reasoning yields that we will never discover the top of a gap by coming across a byte with an AgeStamp more recent than expected (i.e., at variance with Observation 1.c). Indeed, a tainted store operation at $\operatorname{add} r_{1}$ changes PFT(addr $\left.1+1\right)$, which we will encounter first.

\subsubsection{Excess of data}

We now discuss how to determine the beginning of the vulnerable buffer buf, thus we address the problem of unrelated taints. For the sake of simplicity, we again assume that there is no discontinuity at the beginning of buf, i.e., at the point of intrusion detection, buf [0] contains the byte that contributed to the attack.

Consider the successive possible instances of the beginning of the vulnerable buffer. For each of the scenarios we explicitly discuss the contents of essential variables at the time of the overflow and at the time of detecting the intrusion. For the sake of clarity let us denote the memory location of buf [0] by $\operatorname{addr}_{B}$, and the address below buf [0] by $\operatorname{addr}_{A}$. We assume that traversing the memory as discussed above led us to byte $\operatorname{addr} r_{B}$, and we check whether we can draw appropriate conclusions enabling to spot correctly the buffer boundary. 
1. Overflow: PFT $\left(\operatorname{addr}_{B}\right)$ equals 0 ; we set FTS $\left(\operatorname{addr} r_{B}\right)=0$. Detection: We encounter a byte with FTS set to 0 , and we are not inside a discontinuity. Thus we have just encountered the beginning of buf. To make the conclusion clear, note that inside a tainted vulnerable buffer there is only one possibility for a byte to have the FTS indicator unset, namely at the beginning of a gap.

2. Overflow: $\operatorname{PFT}\left(\operatorname{addr}_{B}\right)$ is equal to 1 , but $\operatorname{addr}_{A}$ contains stale data; we set FTS $\left(\operatorname{addr}_{B}\right)$ and unset $\operatorname{PFT}\left(\operatorname{add} r_{B}\right)$. Detection: We encounter a byte with FTS set and PFT unset, which has the stored age stamp of the address beneath it. Observe that since the data at $\operatorname{addr}_{A}$ is stale, AgeStampaddr $A$ is less than the current age stamp, and recall from Listing 2, step i.3 that we will have stored the age stamp in this case. We compare this age stamp with AllocAgeStamp of buf to conclude that at the time of overflow addr ${ }_{A}$ 's value was stale, so we have just encountered the beginning of the vulnerable buffer.

3. Overflow: $\operatorname{PFT}\left(\operatorname{add} r_{B}\right)$ is equal to $1, \operatorname{addr}_{A}$ contains fresh data; we set $\mathrm{FTS}\left(\operatorname{addr}_{B}\right)$ and unset $\mathrm{PFT}\left(\operatorname{addr}_{B}\right)$. Since $\operatorname{addr} r_{A}$ merged with buf together form an area that conforms to all the properties of a crucial region (see Observation 1), we will treat $\operatorname{addr}_{A}$ as a part of the tainted buffer we are looking for. Note that we cannot detect that $\operatorname{addr}_{A}$ belongs to a distinct variable. Most compilers (including gcc) allocate stack memory for a few local variables at once, making it impossible to see the boundaries between successive buffers. Similarly, on the heap, memory is allocated for a structure as a whole, rather than for the individual fields separately. Detection: We come across a byte with FTS set to 1 . Regardless of the existence of the stored age stamp of the memory location below it, we will conclude that at the moment of overflow $\operatorname{addr}_{A}$ 's value was fresh, and so is supposed to belong to the vulnerable buffer. Depending on the application behavior between the moment of overflow and that of detection, we will end up either adding unrelated taints to the crucial tainted memory region or spotting a contradiction with Observations 1-3 and reversing to the last correct byte encountered, $\operatorname{addr}_{B}$. The first possibility comes true only if (a) we reach a buffer that is totally filled with network data, (b) the possible area between this buffer and $\operatorname{addr}_{B}$ appears exactly like an unrelated tainted gap, and (c) additionally, the whole region containing the buffer, the unrelated tainted gap, and the crucial tainted memory region is in accordance with Observations 1-3. Note however, that even in this unlikely case we could only incur false negatives, and never false positives, since the unrelated tainted buffer needs to be filled totally.
We have not discussed what happens if the discontinuity in the vulnerable buffer reaches the buffer's bottom. In principle, the analysis is analogous to the one presented above. What is worth noting, is the fact that we miss part of the crucial tainted memory region, since the bottom part of the vulnerable buffer gets overwritten.

\subsection{Signature Generation}

After the preceding steps have identified the malicious data in memory and generated a one-to-one mapping with bytes in the network trace, we generate signatures capable of identifying polymorphic buffer overflow attacks. Using knowledge about the protocol governing the malicious traffic, we first list the protocol fields including the crucial tainted memory region. Due to possible excess of tainted data in rare scenarios described in Section 4.8, we include a protocol field in a signature either if it contains the violation address, or if a cohesive part of it including at least one boundary can be mapped to the indicated malicious data. We call these fields critical.

Note that vulnerable code usually handles specific protocol fields. Thus, attackers wishing to exploit a certain vulnerability within this code, embed the attack in these protocol fields. If values in such fields contain more bytes than can be accommodated by the buffer, an overflow is sure to occur.

\subsubsection{Vulnerabilities rather than attacks}

We generate signatures for stack and heap overflows by specifying the vulnerability rather than the attack itself. We do so by indicating the protocol fields that should collectively satisfy a condition. In particular, in the current version the signature specifies that the fields should collectively have a length $L$ that does not exceed some maximum, lest they overflow important values in memory. In the simple case with only one protocol field responsible for the attack, $L$ describes the distance between the beginning of the protocol field and the position in the network trace that contains the value that overwrites the target. Otherwise, $L$ is augmented with the lengths of the remaining critical fields. In both cases $L$ is greater or equal to the length of the vulnerable buffer. Signatures can be checked by a protocol dissector (similar to Ethereal) that yields the fields in a flow.

Heap overflows founded on malformed length As mentioned earlier, for heap corruption attempts that manipulate a length field signatures need to relate the critical fields to the length field. Thus, after 
having determined the crucial tainted memory region buf of length $l$, we check in the network trace for the length value $l_{a}$ provided by the attacker. If it is bigger than $l$, we specify that a message contains an attack if the cumulative length of the critical fields is less than $l_{a}$ with the length field greater or equal $l_{a}$. In the second scenario, with $l_{a}<l$, we must be more cautious, since the value provided by the attacker does not need to define the number of bytes, but it could describe amount of integers or any other structures. For now we describe the malicious message similarly as in the case of overflows regarding static-length buffers, requiring conformity of the length value with the actual size of the protocol fields. Thus as a value for $L$ we provide the length field. To assure that the signature is correct we need to verify it by checking whether Prospector spots an illegal operation if we send a message with critical fields filled with arbitrary bytes in the size slightly exceeding length_field. If it appears we are wrong, the only thing we can do is use the semantics of the protocol for a description of the length field.

Multiple fields By handling multiple fields, Prospector fixes and generalizes the signature generation in Covers [16]. Also, unlike Covers, we do not require the protocol dissector to match the granularity in which the application works with protocol messages. The granularity of the dissector may be larger or smaller than that of the application. For instance, the dissector may indicate that a message contains two fields $F 1$ and $F 2$, while the application copies them in one in a single buffer in one go (essentially treating them as a single field $F$ ).

False positives Observe that whenever an application with a given vulnerability receives network data containing the corresponding critical fields with a collective length exceeding $L$ bytes, it will not fit in the application buffer, even if it does not contain any malicious data. Consequently passing it to the application would be inappropriate. In other words, regardless of content, the signatures will not incur false positives in practice. However, in an unlikely scenario it is possible that we cannot correctly determine the crucial tainted memory region, missing a protocol field. This may happen if the gap in crucial tainted memory region reaches the beginning of the buffer, and contains an extra protocol field not encountered before. Notice however, that when we analyze a heap corruption attack which overwrote control data (a red region) on the heap, we will not miss any protocol fields, since the memory dump is performed at the moment of corruption.
Polymorphism By focusing on properties like field length, the signatures are independent of the actual content of the exploit and hence resilient to polymorphism. By focusing on the vulnerabilities, they also detect attacks with different payloads. Such behavior is quite common, especially if part of the payload is stored in the same vulnerable buffer. As the signatures generated by Prospector identify vulnerabilities, they are application specific. As a result, we may generate a signature that causes control flow diversion in a specific version of an application, but there is no guarantee that this is also the case for a different version of the same application. In other words, we need precise information about the software we want to protect.

Value fields The critical fields and the condition that should be satisfied constitute the first, unpolished signature. In practice, however, we may want to characterize more precisely what messages constitute an attack. For instance, when the URL field is the critical field that overflows a buffer in a Webserver, it may be that the overflow only works on GET requests and not for POST requests. In our protocol-specific approach we therefore add a protocol module that determines per protocol which fields may be considered important (e.g., the request type in HTTP) and should therefore be added to the signature. We call such fields value fields as explained in the next section.

Before specifying the signatures, however, we emphasize that making less specific signatures is greatly facilitated when the attack is fingerprinted, i.e., if we know which bytes contributed to the attack. To continue the example, we could simply try to see if the overflow also works for POST request, by crafting a POST message with a similar URL field. We expect much of this process can automated, although we have not yet attempted to do so.

\subsubsection{The final form of Prospector's signatures}

Every signature consists of a sequence of value fields and critical fields. A value field specifies that a field in the protocol should have this specific value. For instance, in the HTTP protocol a value field may specify that the method should be GET for this signature to match, or it could provide the name of a vulnerable Windows .dll. Critical fields, on the other hand, should collectively satisfy some condition. For instance, they should collectively have a length that is less/not less than $L$. We can also put some boundaries on given fields, like in the case of heap overflows based on malformed messages. Example signatures can be found in Section 6.1. 


\subsection{Double-free errors}

We added a module to Prospector to make it deal with double free attacks. Memory managers are sometimes exploited when a programmer makes the mistake of freeing a pointer that was already freed. Doublefree errors do not share the characteristics of heapcorruption attacks in the sense that they do not overflow a buffer, and so when considering the analysis they require special treatment.

Double-free exploits may overwrite any location, resembling the complex heap corruption attacks. Similarly, it is highly probable that when a violation is detected, the memory region that was holding the vulnerable buffer is reused and contains unrelated data. To deal with this issue, whenever free (or realloc) is called, we check for a potential double free error, assuring that the given memory location indeed points to the beginning of an allocated buffer. Otherwise we store the adjacent tainted memory region for possible later use.

\section{Implementation details}

In this section, we discuss main aspects of our implementation of Prospector on Linux using an x86 emulator based on Qemu.

\subsection{Prospector tagging}

To deal with memory tagging Argos introduces a structure similar to page directories in Linux consisting of pagemaps and bytemaps. A pagemap is an array, where each entry corresponds to a bytemap keeping tags for a particular physical page. Here Argos stores all tags on the guest operating system memory, e.g., the network offsets that serve as taint tags. Initially only the pagemap is allocated. Bytemaps are added on demand, when tainted data is copied to a particular physical page for the first time. The network offset tags associated with each byte are 32 bits. To support signature generation we doubled the size of the tag, yielding an additional 32 bits. Of these 32 bits, we designate one bit for the PFT and FTS indicators, one bit for the red marker denoting critical data on the heap, and the remaining 29 bits for the age stamp. We emphasize that age stamps serve only to compare tainted data, so they need only be incremented if a given value was used as a tag to mark tainted data. As most functions and indeed most processes never touch such data, the age stamp may remain untouched. As a result, the age stamp will wrap much more slowly.
Qemu translates all guest instructions to host native instructions by dynamically linking blocks of functions that implement the corresponding operations. With the aim of tracking tainted data being copied to memory we instrument the store function to perform the operations of keeping track of age stamps and setting the extra indicators (PFT and FTS) described in Section 4.5. Here we also check whether the destination memory location is not marked as red (which indicates an overflow and perhaps a complex heap corruption attack, and therefore leads to a dump of the adjacent tainted memory).

\section{$5.2 \quad$ Stale red markers}

As mentioned earlier, to handle complex heap corruption attacks, we mark bytes surrounding allocated chunks of memory as red. If tainted data is written to a red region, this indicates illegal operations which trigger bookkeeping: the memory region is dumped. As we cannot rely on applications releasing all allocated memory, we may end up with stale red markers, possibly leading to unnecessary dumps of memory regions. We describe here how we solve this problem by removing false red indicators.

First of all, we keep counters indicating the number of red markers associated with each physical page in memory. To deal with the problem in the case of pages for the user stack or kernel memory, we monitor new entries added to the TLB as follows. We keep a table of physical pages associated with the identifier of the last process using it. Whenever a new entry corresponding to a kernel address or the user stack is added to the TLB buffer, we check whether the page has a new owner, and if so, we make sure that it does not contain any red markers. If so, we know that neither the user stack nor kernel memory contains the markers.

For the heap we cannot use this method, since dynamically allocated memory can easily be shared between processes, which could remove our markers. Thus, whenever a new buffer is allocated, we assure that its contents do not contain any red regions. First, we check the counter of red markers associated with the given page and, if necessary, clean the memory.

\section{Evaluation}

We evaluate Prospector along two dimensions: effectiveness and performance. While performance is not critical for a honeypot, it needs to be fast enough to generate signatures in a timely fashion. 


\subsection{Effectiveness}

To test our analysis and signature generation, we launched a number of real attacks (as well as handcrafted ones) against Linux on top of Argos. We have not experimented with Microsoft Windows since a small part of the functionality in Prospector is OSspecific, i.e., malloc and free function interposition and (partly) process switch monitoring. For launching attacks, we used the Metasploit framework ${ }^{2}$ and Milw0rm ${ }^{3}$. While we have tested Prospector with many types of attack, in this section we illustrate how Prospector deals with four representative stack- and two heap overflow attacks. These are all real attacks, exploiting real services.

PeerCast Stack Overflow A remote overflow exists in PeerCast v0.1216 and earlier [29]. It fails to perform correct bounds checks on parameters passed in a URL, resulting in a stack-based overflow. An overly long query overwrites EIP stored on the stack. Our analysis engine correctly separated stale data on the stack. A 4-byte discontinuity in the critical tainted memory region was encountered. The final signature follows:

(application: PeerCast, version: v0.1212,
(type: value_field, name: method, value: GET),
(type: critical_field, name: query),
(type: critical_length, value: 476$)$ ).

Subversion Stack Overflow There is a remote overflow in Subversion 1.0.2 [22] which fails to bounds check when calling sscanf() to decode old-styled date strings. In our experiment, an overly long week day overwrites EIP stored on the stack. The resulting signature follows:

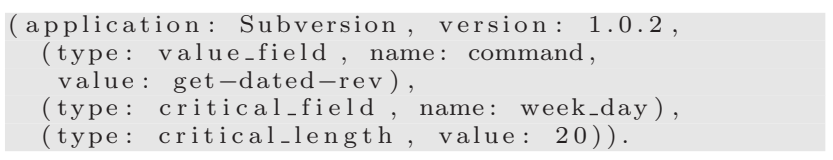

AlsaPlayer Stack Overflow A remote buffer overflow exists in AlsaPlayer 0.99 .76 and earlier [18]. A long "Location" field triggers an overflow in the reconnect function in reader/http/http.c. Our analysis engine encountered a 4-byte discontinuity in the critical tainted memory region. The final signature follows:

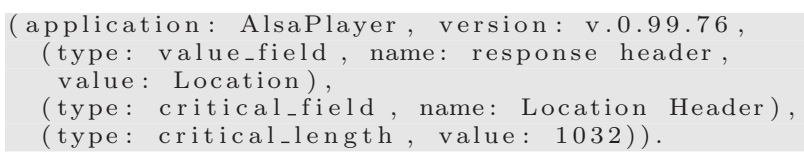

\footnotetext{
${ }^{2}$ The Metasploit Project, http://www .metasploit.com.

${ }^{3}$ Milw0rm, www.milw0rm.com
}

WvTftp Heap Overflow. A heap-based overflow in the WvTftp 0.9 allows remote attackers to execute arbitrary code via a long option string in a TFTP packet [26]. The option name value pairs are given as a NULL terminated option name, followed by an ascii representation of the number value. The function atoi() is used on the value string, and as long as the original part of the string equals a value $>8$ and $<65464$, the string is strcpy'd into the heap buffer. By supplying a long string for the value, the buffer can be overflown. The emulator correctly noticed that the heap control red region was overwritten with network data. The resulting signature follows:

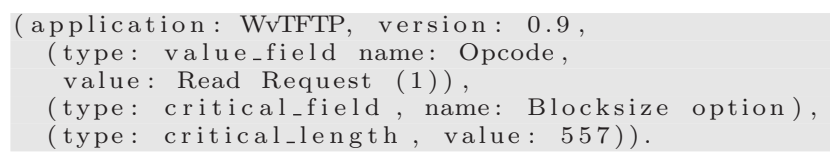

Asterisk Heap Overflow The Asterisk Skinny channel driver for Cisco SCCP phones in v1.0.11 and earlier, v1.2.12 and earlier (chan_skinny.so) incorrectly validates a length value in the packet header. An integer wrap-around leads to a heap overwrite, and arbitrary remote code execution [27]. Asterisk checks whether the inequality (length_value $+8 \leq 1000$ ) holds to convince itself that the user-supplied message fits in the local buffer of size 1000 . Because of the integer wrap, the result of the comparison is positive. And then, the 4 bytes length are copied to the vulnerable buffer, and a read operation is performed storing (length_value +4 ) bytes of the message on the heap. The emulator detects that the control red region on the heap gets overwritten with network data, and dumps the corresponding memory area. In the analysis phase, we first come across the whole SKINNY message but the length field (this part has the same age stamp). Next, we include the 4 bytes underneath it, forming the length, in the crucial tainted memory region (since it is a tainted region with correctly fitting age stamps). Thus the signature specifies the whole SKINNY Packet for Asterisk 1.0.10 not to exceed 1000 bytes. Notice, that even though the length field does not need to be included in the signature, the attack description is still absolutely correct.

libmusicbrainz Stack Overflow. A boundary error within the Download function in lib/http.cpp (v. 2.1.2 and earlier) can be exploited to cause a buffer overflow via a large "Location" field in a HTTP redirection received from a malicious MusicBrainz server [19]. Our analysis engine encountered a 4-byte discontinuity in the critical tainted memory region. The final signature follows:

(application: libmusicbrainz, version: v.2.1.2, (type: value_field, name: response header, 
value: Location ),

(type: critical_field, name: Location Header),

(type: critical_length, value: 73)).

\subsection{Performance}

For realistic performance measurements we compare the speed of code running on Argos and Prospector with that of code running without emulation. Note that while this is an honest way of showing the slowdown incurred by our system, it is not necessarily the most relevant measure. After all, we do not use Prospector as a desktop machine and in practice hardly care whether results appear much less quickly than they would without emulation. The only moment when slowdown becomes an issue is when attackers decide to shun slow hosts, because it might be a honeypot. To the best of our knowledge, automated versions of such attacks do not exist in practice.

Performance evaluation was carried out by comparing the observed slowdown at guests running on top of various configurations of Prospector and unmodified Argos with the original host. The host used during these experiments was an $\operatorname{Intel}(\mathrm{R}) \mathrm{Xeon}(\mathrm{TM}) \mathrm{CPU}$ at $2.8 \mathrm{GHz}$ with $2048 \mathrm{~KB}$ of L2 cache, and $4 \mathrm{~GB}$ of RAM, running Gentoo Linux with kernel 2.6.15.4. The guest OS ran Ubuntu Linux 5.05 with kernel 2.6.12.9, on top of Qemu 0.8, Argos and Prospector. To quantify the observed slowdown we used Apache 2.2.3. We measured its throughput in terms of processed requests per second and the corresponding average response time. We used httperf for generating requests.

Figure 3 shows the results of the evaluation. We tested the benchmark application at the guest running over Argos, and two different configurations of Prospector: both with and without the double free extension module. The graph shows that the achieved throughput increases linearly with the offered load until the server saturates at a load of 48 calls per second in the case of Prospector and 57 for Argos. The response time starts out at about $20-30 \mathrm{~ms}$, and then gradually increases until the server becomes saturated. Beyond this point, response time for successful calls remains largely constant at $3000 \mathrm{~ms}$.

We can conclude that the overhead expressed in throughput of a web server incurred by Prospector compared to Argos is approximately 16\%. We have also performed measurements of slowdown in comparison with the original host (refer to [24] for the full performance evaluation of Argos.) Apache on Argos is about 15 times slower than the one run on the native operating system (on Prospector 18 times). We emphasize that we have not used any of the optimization modules available for Qemu. These modules speed up the emulator to a performance of roughly half that of the native system. While it is likely that we will not quite achieve an equally large speed-up, we are confident that much optimization is still possible. Moreover, even though the performance penalty is large, personal experience with Argos and Prospector has shown us that it is tolerable.

\section{Conclusions}

We have described Prospector, an emulator capable of tracking which bytes contribute to an overflow attack on the heap or stack. By careful analysis, and keeping track of the age of data, we manage to provide such information with greater accuracy than previous approaches while maintaining reasonable performance. The information is important for security experts. We have also used the information to generate signatures for polymorphic attacks by looking at the length of protocol fields, rather than the actual contents. In practice, the number of false positives for the signatures is negligible and the number of false negatives is also low. At the same time, the signatures allow for efficient filters.

\section{References}

[1] P. Akritidis, E. P. Markatos, M. Polychronakis, and K. D. Anagnostakis. Stride: Polymorphic sled detection through instruction sequence analysis. In Proceedings of the 20th IFIP/SEC 2005, 2005.

[2] Anonymous. Once upon a free(). http://doc.bughunter.net/buffer-overflow/free.html.

[3] W. X. S. Bhatkar and R. Sekar. Taint-enhanced policy enforcement: A practical approach to defeat a wide range of attacks. In Proceedings of 15th USENIX Security Symposium, 2006.

[4] D. Brumley, J. Newsome, D. Song, H. Wang, and S. Jha. Towards automatic generation of vulnerability-based signatures. In Proceedings of the 2006 IEEE Symposium on Security and Privacy, May 2006.

[5] R. Chinchani and E. Berg. fast static analysis approach to detect exploit code inside network flows. In In Recent Advances in Intrusion Detection, Seattle, WA, 2005.

[6] M. Christodorescu, S. Jha, S. Seshia, D. Song, and R. Bryant. Semantics-aware malware detection. In Security and Privacy Conference, Oakland, CA, May 2005.

[7] M. Costa, J. Crowcroft, M. Castro, A. Rowstron, L. Zhou, L. Zhang, and P. Barham. Vigilante: end-to-end containment of Internet worms. In Proceedings of the 20th ACM Symposium on Operating Systems Principles, 2005.

[8] Crispin Cowan, Calton Pu, Dave Maier, Heather Hintony, Jonathan Walpole, Peat Bakke, Steve Beattie, Aaron Grier, PerryWagle and Qian Zhang. StackGuard: Automatic Adaptive Detection and Prevention of Buffer-Overflow Attacks. In 7th USENIX Security Symposium, 2002. 

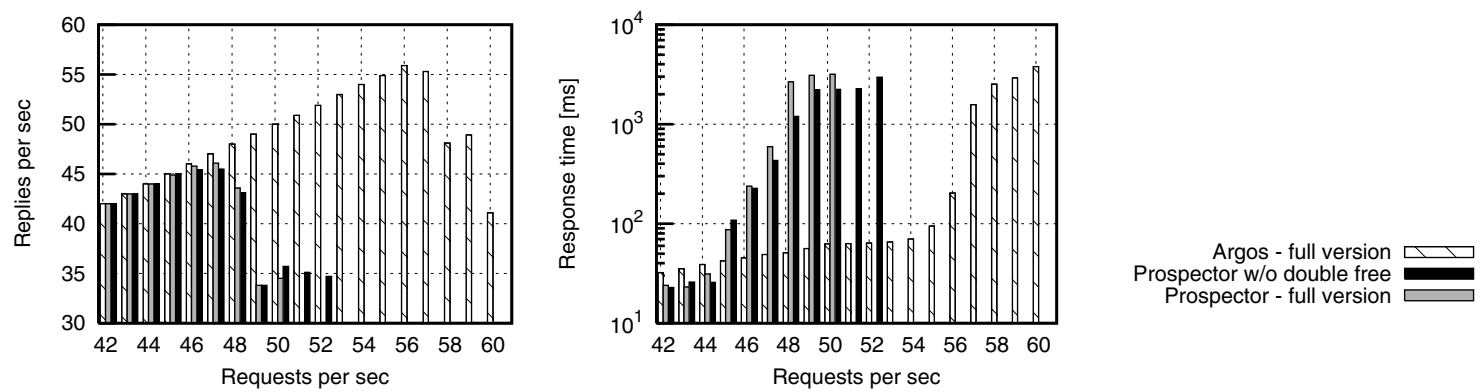

Figure 3. Apache throughput in terms of maximum processed requests per second, and the average response time.

[9] H. Feng, J. Giffin, Y. Huang, S. Jha, W. Lee, and B. Miller. Formalizing sensitivity in static analysis for intrusion detection. In Proceedings the IEEE Symposium on Security and Privacy, Oakland, CA, 2004.

[10] H. Feng, O. Kolesnikov, P. Fogla, W. Lee, and W. Gong. Anomaly detection using call stack information. In Proceedings of the IEEE Security and Privacy Conference, Oakland, CA, 2003.

[11] P. Fogla and W. Lee. Evading network anomaly detection systems: formal reasoning and practical techniques. In Proceedings of the 13th ACM CCS, 2006.

[12] J. T. Giffin, S. Jha, and B. P. Miller. Automated discovery of mimicry attacks. In D. Zamboni and C. Krügel, editors, RAID, volume 4219 of Lecture Notes in Computer Science. Springer, 2006.

[13] C. Krügel, E. Kirda, D. Mutz, W. Robertson, and G. Vigna. Automating mimicry attacks using static binary analysis. In 14 th Usenix Security Symposium, Baltimore, MD, August 2005.

[14] C. Krügel, E. Kirda, D. Mutz, W. Robertson, and G. Vigna. Polymorphic worm detection using structural information of executables. In RAID, Seattle, WA, October 2005

[15] C. Krügel and G. Vigna. Anomaly detection of web-based attacks. In CCS '03: Proceedings of the 10th ACM conference on Computer and communications security, pages 251-261, New York, NY, USA, 2003. ACM Press.

[16] Z. Liang and R. Sekar. Fast and automated generation of attack signatures: a basis for building self-protecting servers. In Proceedings of the 12th ACM conference on Computer and communications security, 2005.

[17] M. V. Mahoney. Network traffic anomaly detection based on packet bytes. In SAC '03: Proceedings of the 2003 ACM symposium on Applied computing, pages 346-350, New York, NY, USA, 2003. ACM Press.

[18] National Vulnerability Database. CVE-20064089 Multiple buffer overflows in AlsaPlayer. http://nvd.nist.gov/nvd.cfm?cvename=CVE-2006-4089, 2006 .

[19] National Vulnerability Database. CVE-20064197 Multiple buffer overflows in libmusicbrainz. http://nvd.nist.gov/nvd.cfm?cvename=CVE-2006-4197, 2006

[20] J. Newsome, B. Karp, and D. X. Song. Polygraph: Automatically generating signatures for polymorphic worms. In IEEE Symposium on Security and Privacy, May 2005.
[21] J. Newsome and D. X. Song. Dynamic taint analysis for automatic detection, analysis, and signature generation of exploits on commodity software. In Proceedings of the NDSS, 2005 .

[22] Open Source Vulnerability Database. Subversion date parsing overflow. http://osvdb.org/displayvuln.php?osvdbid=6301, 2004

[23] R. Perdisci, D. Dagon, W. Lee, P. Fogla, and M. Sharif. Misleading worm signature generators using deliberate noise injection. In Proceedings of the 2006 IEEE Symposium on Security and Privacy (SESP'06), May 2006.

[24] G. Portokalidis, A. Slowinska, and H. Bos. Argos: an emulator for fingerprinting zero-day attacks. In Proc. $A C M$ SIGOPS EUROSYS'2006, Leuven, Belgium, April 2006.

[25] A. Rahbar. Stack overflow on windows vista. http://www.sysdream.com/article.php?story_ id=241\&section_id=77, July 2006.

[26] Secunia. CVE-2004-1636 WvTftp Buffer Overflow Vulnerability, October 2004

[27] SecuriTeam. Asterisk skinny unauthenticated heap overflow, October 2006.

[28] SecurityFocus. CAN-2003-0245 Apache aprpsprintf memory corruption vulnerability. http://www.securityfocus.com/bid/7723/, 2003.

[29] SecurityFocus. CVE-2006-1148 PeerCast Remote Buffer Overflow Vulnerability. http://www.securityfocus.com/bid/17040/info, 2006.

[30] T. Toth and C. Krügel. Accurate buffer overflow detection via abstract payload execution. In Recent Advances in Intrusion Detection, 5th International Symposium, 2002.

[31] US-CERT. Vulnerability notes database. http://www.uscert.gov, 2007.

[32] H. J. Wang, C. Guo, D. R. Simon, and A. Zugenmaier Shield: vulnerability-driven network filters for preventing known vulnerability exploits. SIGCOMM Comput. Commun. Rev., 34(4):193-204, 2004.

[33] K. Wang, G. Cretu, and S. J. Stolfo. Anomalous payloadbased worm detection and signature generation. In Proceedings of the 8th International Symposium on Recent Advances in Intrusion Detection, 2005.

[34] X. Wang, Z. Li, J. Xu, M. K. Reiter, C. Kil, and J. Y. Choi Packet vaccine: black-box exploit detection and signature generation. In Proceedings of the 13th ACM CCS, 2006.

[35] X. Wang, C.-C. Pan, P. Liu, and S. Zh. Sigfree: A signaturefree buffer overflow attack blocker. In Proceedings of 15th USENIX Security Symposium, 2006. 